\title{
Head position affects the antero-posterior location of tapping points
}

\author{
MD. Mahbubur Rahman, Shoji Kohno, Koji Sawada and Yoshiaki Arai \\ Department of Tissue Regeneration and Reconstruction, Division of Removable Prosthodontics, Niigata University \\ Graduate School of Medical and Dental Sciences, Course for Oral Life Science \\ [Recieved: May 10, 2001.]
}

Key Word: Tapping point, Intercuspal position, Central bearing device, Head position, Condylar position

\begin{abstract}
This study assessed how the location of the tapping point is affected by changing the head position. An intra-oral tracing device consisted of a metal tracing plate and a central bearing pin was used in five fully dentate subjects. The tracing plate was fixed to the maxillary part of the device parallel to the occlusal plane. The central bearing pin was fixed to the mandibular part of the device and was located at the midpoint of a line connecting the bilateral first molars. The pin was placed perpendicular to the tracing plate. The appliance was inserted in the mouth and the subject was asked to perform tapping movements with the head Camper and Frankfort plane placed horizontally. The tapping movements $(\mathrm{n}=30)$ were made at a $30 \mathrm{~mm}$-range of motion, and the incisal and condylar points were tracked with a 6-degree-of-freedom jaw movement tracking system (TRIMET; Tokyo Shizaisha Co., Japan).

The antero-posterior distribution of 30 tapping points and their mean position from intercuspal position were analyzed in incisor and condylar points between two head positions. Result revealed that the condylar points corresponding to the tapping points converged into a small area when the head was positioned with the Camper plane horizontal. However, in both head positions, condylar points were located anteriorly from the intercuspal position. From the results, it can be concluded that head position has it's effects on the distribution and mean position of tapping points. In addition, it can be suggested that from the distribution of scattered tapping points, the head should be positioned with the Camper plane horizontal to record the tapping points at a convergent area.
\end{abstract}




\section{Introduction}

The optimum functional occlusion depends mainly on masticatory muscle function, condylar position in the fossa, and the stability of dentition. In the optimum intercuspal position (IP), the masticatory muscle can exert physiological isometric contraction and the condyle may locate in the unstrained stable position in the fossa. When the IP is decayed or lost, we should determine the new IP from the data of condylar position and masticatory muscle function. There are different methods to register the IP. Recording of tapping movement remains popular, because some studies suggested that the convergent points of the tapping movements seems to coincide with the IP and is called muscular position ${ }^{1-4)}$. The muscular position can be controlled mainly by the activity of masticatory muscles and the condylar movement in the glenoid fossa. From this, we can determine the optimum IP as the muscular position from the convergent point of the tapping movement. However the path of the tapping movement is influenced by the head position ${ }^{5-7)}$. frequency of the tapping movement ${ }^{8.9}$ and also the degree of range of jaw motion ${ }^{5.10)}$.

In this study, a new method to record simultaneously the condylar and occlusal positions was developed. We tried the method, by changing the head position to find out the optimum IP as the muscular position is in harmony with the condylar position in the fossa on tapping movements simultaneously with incisor point.

\section{Material \& Methods}

\section{Subject}

Five male adult fully dentate subjects, aged 27-37 years, with normal occlusion volunteered for this study. They had no history of craniomandibular dysfunction. The details of the experimental procedure were thoroughly explained to all subjects but its objective was not disclosed to them.

\section{Registration of tapping movements}

A six-degree-of-freedom measuring system (Tokyo Shizaisha, Co., Tokyo) was used to measure the tapping movements in the incisor and condylar point. The measuring system consists of six high-resolution linear CCD cameras, a control unit, a personal computer for data analysis, a device for digital allocation of reference points, and upper and lower face bows (total weight: $40 \mathrm{~g}$ ) that carry, unilaterally, two small LEDs each side to make up a total of eight.

The face bows are attached onto the buccal surfaces of the

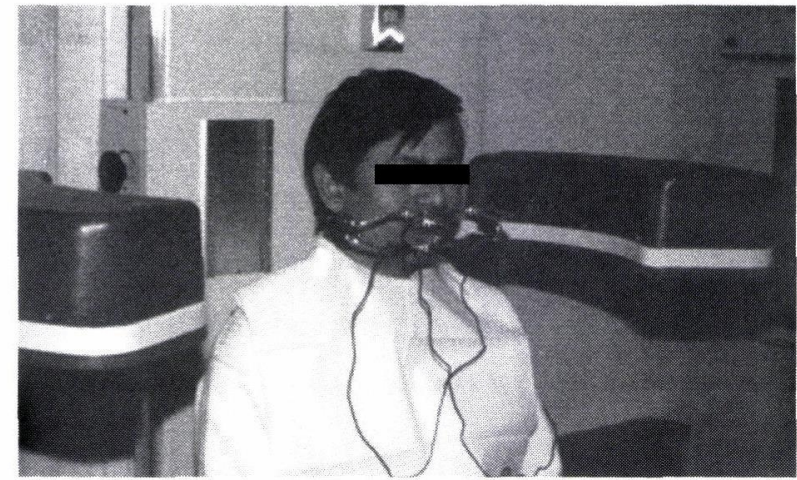

Fig. 1 Subject with attached face bows and the central bearing device in oral cavity was showed for the measurement of jaw movement by TRIMET.

dental arches, and the motion of the LEDs is registered with the six CCD cameras, which are set around the subject's head (Fig. 1). From the data of movements of the LEDs, the movements of the working condyle were calculated by means of analytic geometry. The reference point of condyle was defined as the point $20 \mathrm{~mm}$ medial to the skin on the kinematic axis ${ }^{11.12)}$ point in the right side. Computing system at a rate of 100 points per second with an overall accuracy of 0.15 $\mathrm{mm}$ processed the data.

\section{Experimental Procedure}

The subject was comfortably seated upright with back support on a dental chair. The head was free from any restriction and positioned with the Camper and Frankfort plane horizontal.

The intra-oral tracing device was inserted on the dental arches and the tapping movements terminated in contact with tracing pin and tracing plate of intra-oral device without any contact of the dentition. The intra-oral tracing device consisted of a metal tracing plate and a central bearing pin. The tracing plate was fixed on the upper dental arch parallel to the occlusal plane. The central bearing pin was located at the midpoint of a line connecting the bilateral first molars of the lower dental arch and was made perpendicular to the tracing plate (Fig. 2).

In this study the subjects were instructed to perform tapping movements with a $30 \mathrm{~mm}$ range of motion at a frequency of $2 \mathrm{~Hz}$. for 15 seconds. Tapping movements were preceded by 2 to 3 practice attempts before the actual recording. 


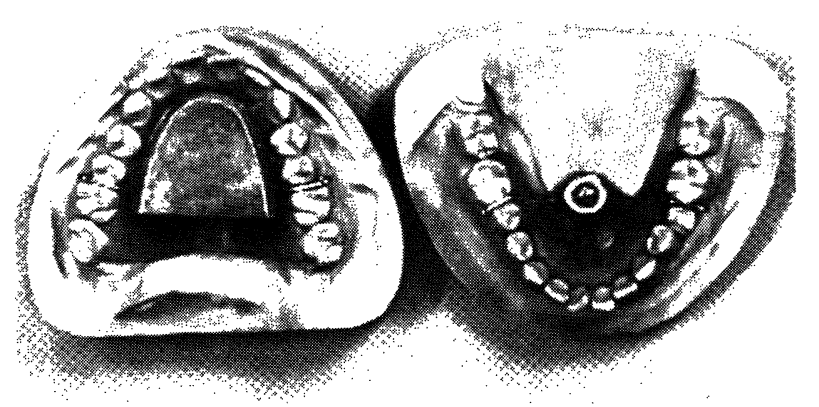

Fig. 2 The central bearing device on working model was used for this study.

The subjects were asked to do the tapping movements for three consecutive trails. The jaw movements were performed following the sound signals of a metronome.

\section{Analyzing method}

The data were analyzed in the sagittal projection viewed from the incisal and right condylar points of the subject.

In this study, we used the intraoral central bearing device and the vertical dimension was increased to record the tapping movements without contact of dentition. Therefore, the true IP of the dentition was lost on the tracing plate. Then, the geometrically identical point of the IP was obtained on the Cartesian coordinates of the incisal point by means of only rotation of the condyle in the same position of the IP in the mandibular fossa with some degree of opening of the mouth. The position can be calculated as an intersection of the horizontal coordinate of the incisal point and the arch around the kinematic axis in the condyle ${ }^{11.12)}$, which is located in the same position as the IP. We denoted this point as calculated IP.

Ten tapping movements were selected from the middle of each of the three trails. So, we evaluated the location of the convergent point and their standard deviation (SD) of the 30 tapping movements antero-posteriorly. The reference positions were the calculated IP at the incisal point and the condyle position in the IP at the condylar point.

\section{Results}

\section{Convergence of tapping points}

Figure 3 demonstrates the effect of head position on the convergence of tapping points in one subject. The figure
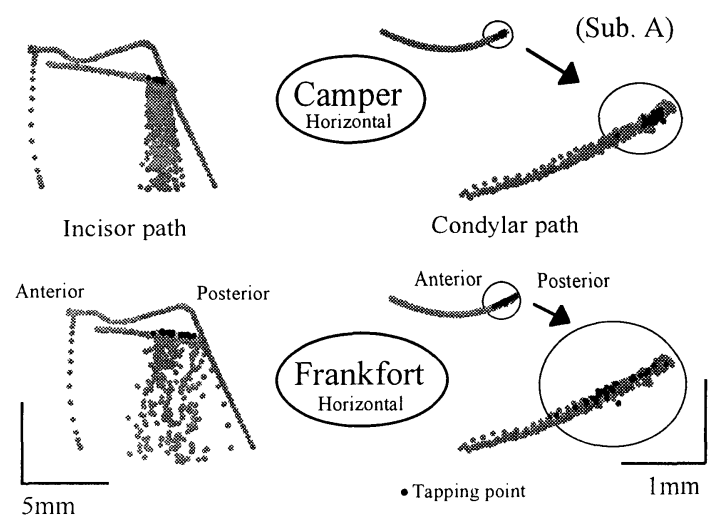

Fig. 3 Effects of head position on the convergence of tapping points $(n=30)$, were demonstrated in incisor point (left side) and the corresponding position of condylar points (right side) were superimposed in sagittal border movement.

shows clearly that in the head position with Camper plane horizontal, the tapping points became convergent and the corresponding position of the condyle also coincided in a small area than in the head position with Frankfort plane horizontal.

Tapping points should be convergent to determine the jaw position for bite registration. So, we obtained the standard deviation of thirty tapping points to assess the degree of the convergence of tapping movements on each head position. The standard deviation of thirty tapping points of five subjects are shown in Fig. 4 and Table 1. The standard deviation of tapping points in the incisor point ranged from $0.13 \mathrm{~mm}$ to $1.01 \mathrm{~mm}$ with a mean of $0.49 \mathrm{~mm}$ in the head position with Frankfort plane horizontal. When the head position was changed to Camper plane horizontal, the standard deviation of tapping points became smaller and ranged from $0.17 \mathrm{~mm}$ to $0.54 \mathrm{~mm}$ with a mean of $0.31 \mathrm{~mm}$. In incisor point with the head position camper plane horizontal, the standard deviation of tapping points became smaller in case of subjects A and B than with the head position Frankfort plane horizontal. These results however were reversed with subjects $\mathrm{C}, \mathrm{D}$ and $\mathrm{E}$. But the absolute values of the standard deviation in these three subjects were very small.

We measured the deviation of the condylar points on the tapping points of thirty tapping movements in five subjects. In the head position with Frankfort plane horizontal, the standard deviation ranged from $0.1 \mathrm{~mm}$ to $0.9 \mathrm{~mm}$ with a mean of 


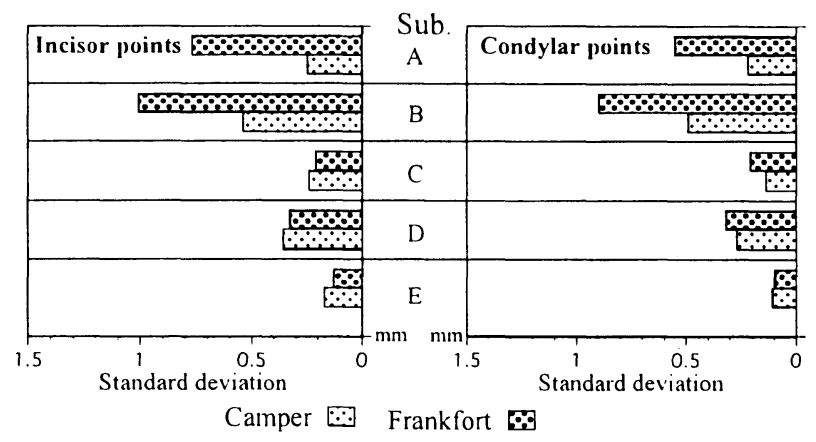

Fig. 4 Difference of the convergence of tapping points represented by the antero-posterior standard deviation of tapping points $(n=30)$, in two head positions were demonstrated in incisor point (left side) and condylar points (right side).
Table 1 Antero-posterior standard deviation of the tapping points $(n=30)$, from the calculated IP in incisor point and the real IP in condylar point.

\begin{tabular}{ccccc}
\hline \hline $\begin{array}{c}\text { Head position } \\
\text { Subjects }\end{array}$ & \multicolumn{2}{c}{ Frankfort horizontal } & \multicolumn{2}{c}{ Camper horizontal } \\
incisor & condyle & incisor & condyle \\
\hline A & 0.77 & 0.55 & 0.25 & 0.22 \\
B & 1.01 & 0.90 & 0.54 & 0.49 \\
C & 0.21 & 0.21 & 0.24 & 0.14 \\
D & 0.33 & 0.32 & 0.36 & 0.27 \\
E & 0.13 & 0.10 & 0.17 & 0.11 \\
\hline Mean & 0.49 & 0.42 & 0.31 & 0.25 \\
\hline
\end{tabular}

point in the fossa from the real IP in five subjects (Fig. 5 and Table 2).

In the incisor point, the mean position of thirty tapping points were $0.67 \mathrm{~mm}$ anterior with a range of $0.1 \mathrm{~mm}$ to 1.38 $\mathrm{mm}$ from the calculated IP in the head position with Frankfort plane horizontal. When the head position was changed to Camper plane horizontal, the mean position of tapping points became nearer $0.46 \mathrm{~mm}$ with a range of $0.23 \mathrm{~mm}$ to $0.62 \mathrm{~mm}$ anterior to the calculated IP.

We also obtained the mean position of the condylar point in each tapping point of thirty tapping movements in five sub-

Table 2 Antero-posterior mean position of the tapping points $(n=30)$, from the calculated IP in incisor point and the real IP in condylar point

\begin{tabular}{ccccc}
\hline \hline Head position & \multicolumn{2}{c}{ Frankfort horizontal } & \multicolumn{2}{c}{ Camper horizontal } \\
Subjects & incisor & condyle & incisor & condyle \\
\hline A & 1.38 & 0.78 & 0.23 & 0.19 \\
B & 1.15 & 0.92 & 0.57 & 0.54 \\
C & 0.10 & 0.17 & 0.42 & 0.12 \\
D & 0.34 & 0.34 & 0.47 & 0.51 \\
E & 0.40 & 0.48 & 0.62 & 0.56 \\
\hline Mean & 0.67 & 0.54 & 0.46 & 0.38 \\
\hline
\end{tabular}


jects. It was $0.54 \mathrm{~mm}$ anterior with a range of $0.17 \mathrm{~mm}$ to $0.92 \mathrm{~mm}$ from the real IP in the head position with Frankfort plane horizontal. When the head position was changed to Camper plane horizontal, the mean position of the condylar point became nearer $0.38 \mathrm{~mm}$ with a range of $0.12 \mathrm{~mm}$ to $0.56 \mathrm{~mm}$ anterior to the real IP.

Data revealed that in all subjects, the tapping points did not coincide with the IP, but were located anterior to it.

\section{Discussion}

\section{Aim of the experiment}

To determine optimum occlusal position in harmony with the musculoskeletally stable position of the joint, correct registration of jaw relation is essential in clinical practice. Current studies suggested that the masticatory muscles function more harmoniously when the condyles are in stable position in the fossa and at that time the teeth are in intercuspation. Furthermore, it is thought that the intercuspal position coincide with the muscular position, and the muscular position can be obtained from convergent point of the tapping movement. However, the path of tapping movement is influenced by the head position, tapping frequency and the tapping range of motion.

In this study, we considered the tapping movement points as the reference position and designed our experiment by changing the head position so that the registration of tapping movements can be obtain in or near to the IP at a convergent area.

\section{Methodology}

Usually, the jaw position is evaluated from the data of incisor points. But when the teeth are lost, the jaw position is guided by the condyle and the masticatory muscle function. Therefore, it is necessary to determine the jaw position from the data of occlusal area and the condylar position. In this study, we used the central bearing device and registered the tapping points in the head position with Camper and Frankfort plane horizontal and described our data in both incisor and condylar point to find out the condylar position in the fossa corresponding to the tapping movements in incisor point. In the study of K. Ikeda ${ }^{5)}$ reported that the highest rotation value of condyle obtained it's stable position in the fossa when the tapping movements were recorded with a frequency of $3 \mathrm{~Hz}$. at an interincisal distance of $30 \mathrm{~mm}$ with the head's Camper plane horizontal. In our study we also followed the same cri- teria of tapping movement except the frequency. As we used the central bearing device in the oral cavity, the tapping strokes on the tracing plate elongated the time of contact phase which was inconvenient for the subjects to perform tapping movements by $3 \mathrm{~Hz}$. So, we reduced the tapping frequency to $2 \mathrm{~Hz}$. for the smoothness of the movements ${ }^{8}$.

\section{Result of the experiment}

The results of our experiment suggested that when the head was positioned so that its Frankfort plane horizontal, the standard deviation of condylar points observed from thirty tapping points were scattered and ranged from $0.1 \mathrm{~mm}$ to $0.9 \mathrm{~mm}$. However, when the head position was changed to Camper plane horizontal, the standard deviation became smaller by about $60 \%, 46 \%, 33 \%$ and $16 \%$ in case of subjects A, B, C and D. But the distribution increased by about $10 \%$ with subject E. However, this increased distribution was less in comparison to other subjects in the head position with Camper plane horizontal.

It indicates that the tapping points in the head position with Camper plane horizontal showed more convergence than in the head position with Frankfort plane horizontal. From this result, it can be concluded that from the distribution of scattered tapping points, head position should be placed with the Camper plane horizontal to record the tapping points at a convergent area.

In the head position with Frankfort plane horizontal, the condyle translated anteriorly from its IP during the thirty tapping movements. In the five subjects, the mean position of the condyle corresponding to the tapping movements ranged from a distance of $0.17 \mathrm{~mm}$ to $0.92 \mathrm{~mm}$ with a mean of 0.54 $\mathrm{mm}$. Whereas, when the head position was changed to Camper plane horizontal, the condyle points were located a little anterior from the IP and ranged from $0.12 \mathrm{~mm}$ to 0.56 $\mathrm{mm}$ with a mean of $0.38 \mathrm{~mm}$. The distance of the condylar position to the IP reduced by about $76 \%, 41 \%$ and $29 \%$ with subjects A, B and C. And with subjects D and E, the distance increased by about $50 \%$ and $17 \%$ accordingly. However, these increased distances were less in comparison to subjects $\mathrm{A}$ and $\mathrm{B}$, in the head position with Frankfort plane horizontal.

In case of subjects $\mathrm{A}$ and $\mathrm{C}$, with the head position Camper plane horizontal, the condylar points mean location corresponding to the tapping movements were $0.19 \mathrm{~mm}$ and 0.12 $\mathrm{mm}$ anterior to the IP. This result supports the definition of stable condylar position in the fossa ${ }^{13)}$. In subject $B$, the tap- 
ping points mean location was $0.54 \mathrm{~mm}$ anterior to the IP, which is higher than the range of the stable position of the condyle in the fossa. However, this distance was about half to the tapping points mean location with the head position Frankfort plane horizontal.

In this study tapping points in both head positions were located anterior to the IP. K. Ikeda ${ }^{5)}$ reported that the condyle located the position of the IP observed from the tapping movement points in the head position with Camper plane horizontal. But, in this experiment the authors observed that in both head positions the tapping points were located anterior rather than coincides the IP. It maybe due to the intraoral tracing device used and the tracing plate of the device placed parallel to the occlusal plane. This position is not perpendicular to the jaw closing path ${ }^{14)}$. However, the limitation of the design of the tracing device was unable to inhibit the protrusive movement of the jaw during jaw tapping movements, thereby may produced negative effect on the data of tapping movements. Therefore, it provides us to extend our thinking on the inclination of tracing plate in future study.

\section{References}

1) Brill, N., Lammie, G.A., Osborn, J. et al.: Mandibular positions and mandibular movements, Brit dent J, 106: 391-400, 1959.

2 ) Brill, N. and Tryde, G.: Physiology of Mandibular Positions, Front Oral Physiol, 1: 199-237, 1974.

3 ) Fujii, H. and H. Mitani.: Reflex response of the masseter and temporal muscles in man, J dental Res, St. Louis, 52 : 1046-1050, 1973.

4 ) Moller, E.: The chewing apparatus, Acta Physiologica Scandinavica, 69: supplement 280, 1966.

5 ) Ikeda, K., Kohno, S., Tsuchida, Y. et al.: Method to determine an ideal maxillomandibular relationship from tapping movements using as reference the most stable position of condyle, J Jpn Prosth Soc, 40: 964-971, 1996. (Japanese with english abstract)

6 ) Hayashi, H.: Studies on the antero-posterior tooth contact position through small opening and closing movement of the mandible under various kinds of posture and opening-closing condition, The journal of the Tokyo dental college society, 80: 1-31, 1980. (Japanese)

7 ) Goldstein, D.F., Kraus, S.L. and Williams, W.B.: Influence of cervical posture on mandibular movement, $\mathrm{J}$ Prosthet Dent, 52: 421-426, 1984.

8 ) Naeije, M. and Honee, G.L.J.M.: The reproducibility of movement parameters of the empty open-close-clench cycle in man and their dependency on the frequency of movements, J Oral Rehabil, 6: 405-415, 1979.

9 ) Widmalm, S.E. and Hedegard, B.: Kinematics of the tooth tapping movement, J Oral Rehabil, 4: 237-246, 1977.

10) Kawaguchi, T.: A study of tooth contact positions during habitual closures and swallowing movements with new electric measuring apparatus, J Jpn Prosth Soc, 12: 398-423, 1968. (Japanese with english abstract)

11) Kohno, S. and Ishiwara, T.: Analysis of movement of mandibular condyle in sagittal plane, Bulletin of Tokyo Medical and Dental Univ, 18: 233-245, 1971.

12) Kohno, S.: Analyse der kondylenbewegung in der sagittalebene, Dtsch Zahnarztl Z, 27: 739-743, 1972. (German)

13) Oishi, T.: A study on the anatomical structure of temporomandibular joint from the standpoint of mandibular movement, J Jpn prosth Soc, 11: 197-220, 1967. (Japanese with english abstract)

14) Ogawa, T., Koyano, K. and Suetsugu, T.: The relationship between inclination of the occlusal plane and jaw closing path, J Prosthet Dent, 76: 576-580, 1996. 